\title{
Distribución geográfica del mono aullador rojo (Alouatta seniculus) y la fiebre amarilla en Colombia
}

\author{
Juan Piedrahita-Cortés, Diego Soler-Tovar \\ Grupo de Epidemiología y Salud Pública, Universidad de La Salle, Bogotá, D.C., Colombia
}

\begin{abstract}
Introducción. Colombia es un país con gran diversidad de primates no humanos, entre los cuales se destaca el mono aullador rojo (Alouatta seniculus) por su distribución y el papel que desempeña en la presentación de la fiebre amarilla.

Objetivo. Describir la coincidencia geográfica del hábitat del mono aullador rojo y la presencia de fiebre amarilla.

Materiales y métodos. Se hizo un estudio de carácter descriptivo. Los antecedentes de la fiebre amarilla en Colombia se obtuvieron de los informes y boletines del Instituto Nacional de Salud y del estudio de 2013 de Segura, et al. La presencia de $A$. seniculus se determinó con base en la plataforma Global Biodiversity Information Facility y el Sistema de Información sobre Biodiversidad de Colombia; los mapas se elaboraron con el programa Diva-Gis, y el modelo de nicho ecológico bajo las condiciones actuales, con el programa Maxent.

Resultados. Los departamentos con mayor presencia de $A$. seniculus fueron Antioquia, Meta y Casanare; en $69,5 \%$ de los departamentos con antecedentes de notificación de fiebre amarilla también había A. seniculus. El modelo de nicho ecológico evidenció que Antioquia, Bolívar, La Guajira, Magdalena, Meta, Santander, Norte de Santander y Vichada tenían porciones de territorio con un índice de probabilidad cercano a 0,9 (90\%).

Conclusiones. En 69,5\% de los departamentos con antecedentes de fiebre amarilla se registró la presencia de $A$. seniculus, lo cual resulta relevante por el papel que los primates no humanos desempeñan como reservorio natural del virus y por su contribución en la presentación de la fiebre amarilla, lo cual les confiere gran utilidad como centinelas.
\end{abstract}

Palabras clave: Alouatta, enfermedades de los primates, fiebre amarilla, epidemiología, Flavivirus, medicina tropical, zoonosis, América Latina.

doi: http://dx.doi.org/10.7705/biomedica.v36i0.2929

\section{Geographical distribution of the red howler monkey (Alouatta seniculus) and yellow fever in Colombia}

Introduction: Colombia is a country with an important diversity of non-human primates, of which the red howler monkey (Alouatta seniculus) stands out because of its distribution and the role it plays in the occurrence of yellow fever.

Objective: To describe the geographic co-occurrence of Alouatta seniculus and the reported presence of yellow fever.

Materials and methods: We conducted a descriptive study. The reported presence of yellow fever in Colombia was obtained from the reports and bulletins issued by the Instituto Nacional de Salud, and the study by Segura, et al. (2013). The occurrence of $A$. seniculus was determined based on the data from the Global Biodiversity Information Facility and the Colombian Biodiversity Information System. A map of the occurrence was developed using the DIVA-GIS program, and the ecological niche model under current conditions was created with the Maxent program.

Results: The departments with the highest occurrence of $A$. seniculus were Antioquia, Meta and Casanare; $69.5 \%$ of the departments with reported history of yellow fever had co-occurrence with $A$. seniculus. The ecological niche model showed that Antioquia, Bolívar, La Guajira, Magdalena, Meta, Santander, Norte de Santander and Vichada had geographical portions with a probability rate nearing to $0.9(90 \%)$.

\section{Contribución de los autores:}

Juan Piedrahita-Cortés: revisión de literatura y escritura del manuscrito

Diego Soler-Tovar: diseño de mapas en Diva-Gis y modelos de nicho ecológico en Maxent, diseño y contribución a la estructuración del manuscrito

Todos los autores contribuyeron a la concepción de la idea de investigación, a la interpretación de los resultados y la redacción del manuscrito. 
Conclusions: In $69.5 \%$ of the departments with a history of yellow fever there was co-occurrence with $A$. seniculus, which is relevant because non-human primates play a well-known role as natural reservoirs of the virus, and they might contribute to the occurrence of the yellow fever, which makes them very useful as sentinels.

Key words: Alouatta, primate diseases, yellow fever, epidemiology, Flavivirus, tropical medicine, zoonoses, Latin America.

doi: http://dx.doi.org/10.7705/biomedica.v36i0.2929

Colombia es un país con una notable biodiversidad cuyo gran número de especies de fauna y flora se atribuye a su geografía ecuatorial y a su compleja topografía. El país es el cuarto más diverso del mundo en mamíferos registrados, con cerca de 471 especies nativas confirmadas. Es uno de los países más diversos del mundo en especies de primates junto con Brasil, Zaire, Camerún, Indonesia, Madagascar y Perú, pues cuenta con 31 especies y múltiples subespecies (1).

En este estudio la atención se centró en la especie Alouatta seniculus, por ser la de mayor distribución en el país, así como por su papel de huésped o reservorio del virus de la fiebre amarilla (1-3) (figura 1). La fiebre amarilla es una enfermedad viral aguda, producida por un arbovirus del género Flavivirus, familia Flaviviridae, que además es zoonótica, endémica y con el riesgo de reemerger en seres humanos, pese a que existe vacuna, por lo cual se la considera de interés en salud pública $(2,4,5)$.

Entre las condiciones que influyen en su aparición se destacan la amplia distribución geográfica de los vectores (Aedes aegypti, Aedes albopictus, Haemogogus janthinomys y Sabethes sp.), la deforestación, el crecimiento demográfico, la cobertura de la vacunación, los perfiles ocupacionales y la variabilidad climática, los cuales afectan la distribución del agente, del huésped y del vector (figura 2). A estos factores se suman, en Colombia, el desplazamiento forzado, la explotación de zonas selváticas vírgenes, la violencia y el narcotráfico, factores que complican la situación $(4,6,7)$. La fiebre amarilla se combate con la vacunación y con adecuadas medidas de vigilancia (8).

\author{
Correspondencia: \\ Juan Piedrahita-Cortés, Carrera 7 № 172-85, Programa de \\ Medicina Veterinaria, Universidad de La Salle, Bogotá, D.C., \\ Colombia \\ Teléfono: (317) 3754711 \\ jmpcvet@gmail.com \\ Recibido: 30/06/15; aceptado: 10/02/16
}

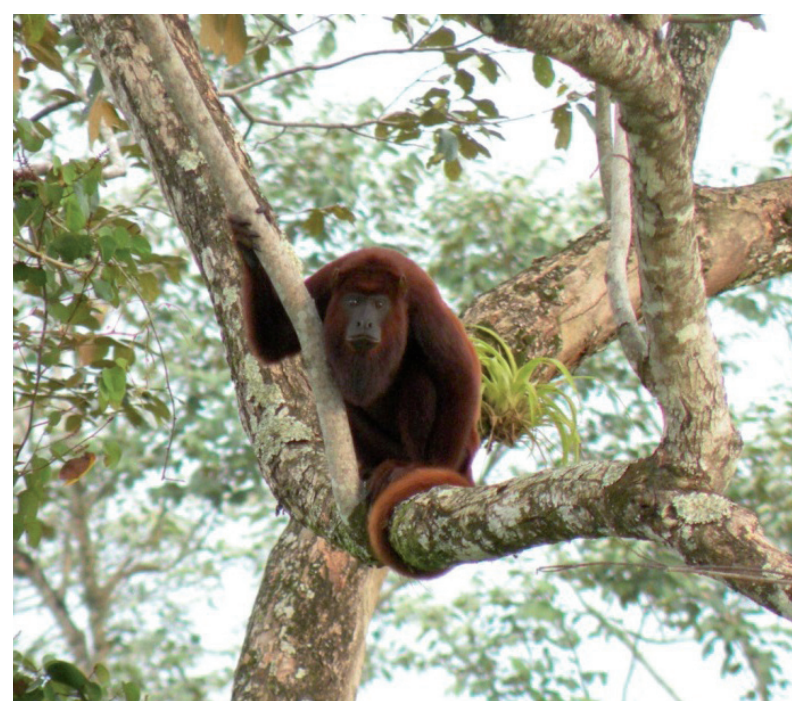

Figura 1. Mono aullador rojo (castaño) (Alouatta seniculus)

En el marco de las políticas frente a la enfermedad, en el país se han expedido los decretos 1693 de 1979, 2257 de 1986, 786 de 1990, y 2323 y 3518 de 2006, además de la Resolución 412 de 2000 y la adopción de lo dispuesto en el Reglamento Sanitario Internacional de 2005; en dichos decretos se establecen las obligaciones y las competencias frente a la enfermedad, pero en ninguno de estos instrumentos legales se reglamentan las medidas relacionadas con la presencia de los primates no humanos. Se sabe, además, que el tráfico de fauna y la interacción con los reservorios pueden representar un riesgo para los humanos. Por otro lado, la fiebre amarilla es una enfermedad de aparición cíclica que suele presentarse cada cinco o siete años y se sabe que hay circulación activa del virus selvático, característica que la hace más impredecible (9-11).

El objetivo del presente artículo fue describir la presencia simultánea de $A$. seniculus, establecida según la información disponible en la Global Biodiversity Information Facility (GBIF) y en el Sistema de Información sobre Biodiversidad de Colombia (SiB Colombia), y de antecedentes reportados de fiebre amarilla en Colombia. 

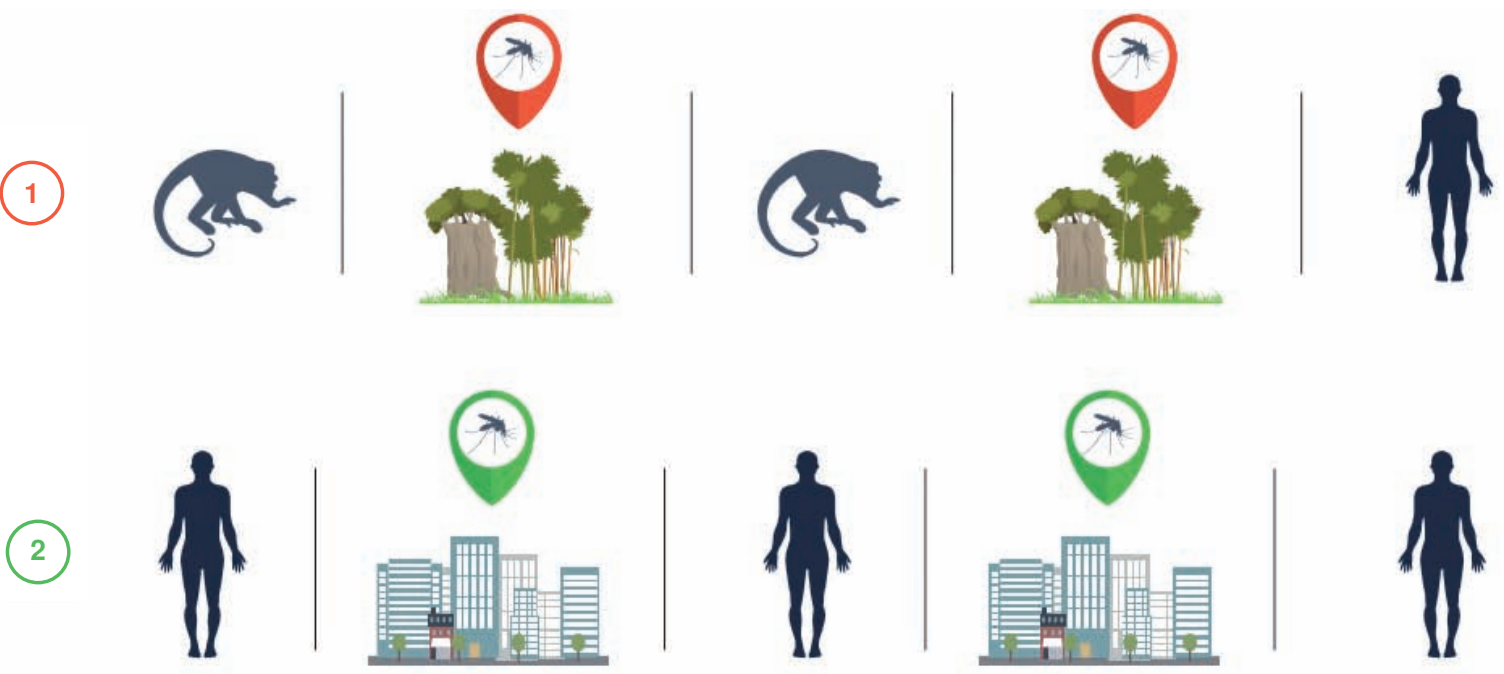

Figura 2. Ciclos de transmisión de la fiebre amarilla. 1: Ciclo de transmisión selvático; 2: Ciclo de transmisión urbano llustración de David Mauricio Suárez

\section{Materiales y métodos}

Se hizo un estudio de carácter descriptivo. Los antecedentes de la presencia de fiebre amarilla en Colombia se obtuvieron de los informes y boletines del Instituto Nacional de Salud y de otras publicaciones, primordialmente del informe de fiebre amarilla en el cuarto periodo epidemiológico de 2015, y del artículo "Tendencias de la mortalidad por fiebre amarilla, Colombia, 1998-2009", publicado por Segura, et al., en el 2013 (12).

La presencia de $A$. seniculus se determinó a partir de los datos de georreferenciación disponibles en la Global Biodiversity Information Facility (GBIF http://www.gbif.org) y en el Sistema de Información sobre Biodiversidad de Colombia (SiB Colombia - http://www.sibcolombia.net/web/sib/home); con esta información, se elaboró el mapa de los sitios donde se encuentra la especie en Colombia usando el programa Diva-Gis (http://www.diva-gis.org), el cual es de libre acceso, no comercial, de fácil manejo para el mapeo y análisis de la información espacial, y de mucha utilidad en el estudio de la distribución de organismos para explicar patrones geográficos y ecológicos. Se elaboró el modelo de nicho ecológico bajo las condiciones actuales con el programa de modelado de máxima entropía Maxent (http://www.cs.princeton.edu/ schapire/ maxent/), utilizando 19 capas bioclimáticas en una resolución de 2,5 arc-minutos (www.worldclim.org).

Para la búsqueda de la literatura científica, se emplearon los siguientes términos de los Descriptores en Ciencias de la Salud (DeCS):
("Yellow Fever" OR "Fiebre Amarilla") AND ("Primates" OR "Alouatta") AND ("Colombia" OR "America"). Las bases de datos y los sistemas de índices y resúmenes (SIRes) utilizados fueron: Bireme, Doaj, Lilacs, Pubmed, Redalyc, Scielo, Science Direct y Scopus.

\section{Resultados}

A continuación se presentan los mapas de la distribución de $A$. seniculus en Colombia según la GBIF y el SiB Colombia (figura 3), en los cuales se observa que los departamentos con mayor número de registros para la especie son Antioquia, Meta y Casanare. Por otro lado, se presenta el modelo de nicho ecológico bajo las condiciones actuales (figura 4), en el cual se plantea un índice de probabilidad de 0 a 1 para la presencia de la especie en estudio; en el modelo se destacan Antioquia, Bolívar, La Guajira, Magdalena, Meta, Santander, Norte de Santander y Vichada como los departamentos con zonas geográficas cuyo índice de probabilidad para la presencia de $A$. seniculus es cercano a 0,9 (90\%), y Atlántico, Boyacá, Casanare, Cesar, Córdoba, Sucre y Risaralda, como aquellos con zonas geográficas con un índice de probabilidad cercano a 0,7 (70 \%), el cual se basa en los 19 factores bioclimáticos determinantes de la presencia del mono aullador rojo; como se ve, la mayor favorabilidad para la presencia de la especie se da en la región andina.

En el mapa de los casos confirmados de fiebre amarilla a nivel municipal (figura 5) reportados en el informe del Instituto Nacional de Salud para 


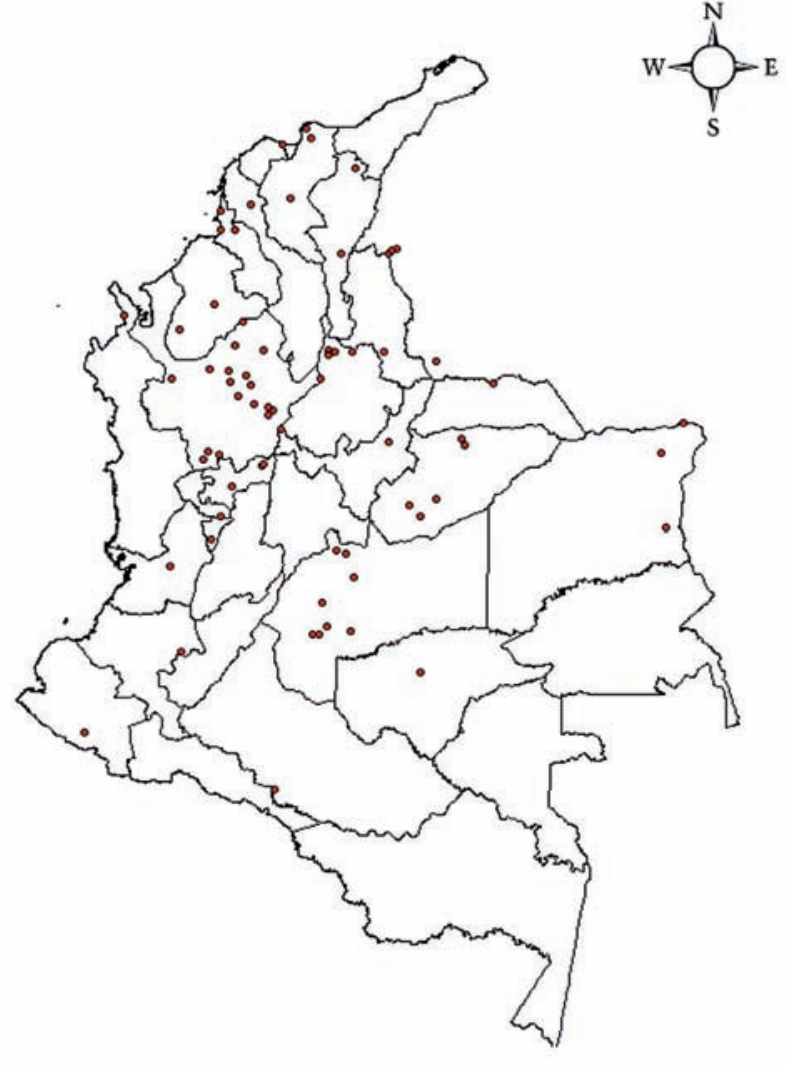

Figura 3. Localidades (puntos rojos) con presencia de Alouatta seniculus. Se destacan Antioquia, Meta y Casanare (modelado con el programa Diva-Gis)

Fuente: SiB Colombia y GIBF.org (27 de febrero de 2015, GBIF Occurrence - http://doi.org/10.15468/dl.94tw36)

el cuarto periodo epidemiológico de 2015, se observa que las regiones Caribe y de la Orinoquia fueron las más afectadas. Según Segura, et al., los departamentos con antecedentes de casos probables o confirmados de fiebre amarilla de 2006 a 2009, así como de mortalidad entre 1998 y 2009, fueron 23, incluido el distrito de Bogotá, lo cual se correlacionó con la presencia geográfica de $A$. seniculus según la GBIF y el SiB Colombia (cuadro 1) (12).

En $69,5 \%$ de los departamentos con antecedentes de fiebre amarilla existe el mono aullador rojo, según los registros de la GBIF y el SiB Colombia, y en $30,5 \%$ no había registro de su presencia. Por otro lado, en siete departamentos había $A$. seniculus, pero no existían antecedentes de fiebre amarilla.

Se evidenció que en $50 \%$ de los 32 departamentos de Colombia había simultáneamente antecedentes de fiebre amarilla y monos aulladores rojos.

\section{Discusión}

Este es un análisis básico que no permitió establecer de manera concluyente la existencia de primates no humanos en las áreas con antecedentes de casos de fiebre amarilla, pero, con base en la evidencia científica disponible en torno al papel que estos desempeñan como reservorios del virus y los antecedentes de epizootias en investigaciones de casos de fiebre amarilla, es probable que dichas variables tengan una correlación significativa. La participación del mono aullador rojo en el ciclo de transmisión del agente de la fiebre amarilla selvática no debe generar rechazo de los primates no humanos en el territorio nacional, por el contrario, ello debe fomentar su estudio y la adopción de medidas de protección para aprovecharlos como centinelas de la enfermedad en Colombia $(2,4,5)$.

La plataforma GBIF ha sido desarrollada por una organización intergubernamental que integra 53 países y 43 organizaciones internacionales. Funciona como una red de nodos nacionales de acceso libre a los datos de biodiversidad de todo el mundo para apoyar la investigación científica, fomentar la conservación biológica y favorecer el desarrollo sostenible; el SiB Colombia es una red informática de acceso libre que facilita herramientas para la integración, publicación y consulta de la información sobre biodiversidad. Sin embargo, el registro de $A$. seniculus está sesgado por el número de registros para la especie en Colombia. Es necesario anotar que el hecho de que no haya registros de su presencia puede estar relacionado con la cantidad de investigaciones $\mathrm{y}$, en consecuencia, el número de localidades registradas en una y otra plataforma.

Este sistema es útil porque no existe un conocimiento completo sobre la tendencia, la distribución o los antecedentes de la fiebre amarilla en los reservorios y puede proveer una oportunidad para incentivar las investigaciones en salud en asociación con redes y organizaciones de biodiversidad en el mundo. Para el caso de Colombia, no existe un estudio consolidado sobre antecedentes de la fiebre amarilla en los reservorios, pero sí existen anotaciones sobre epizootias en algunos documentos independientes (cuadro 2). Otros autores, como Defler, han llevado a cabo estudios sobre la distribución de diversos primates en Colombia, los cuales son muy útiles desde el punto de vista biológico porque detallan el tipo de ambientes y de climas de algunas de las regiones 


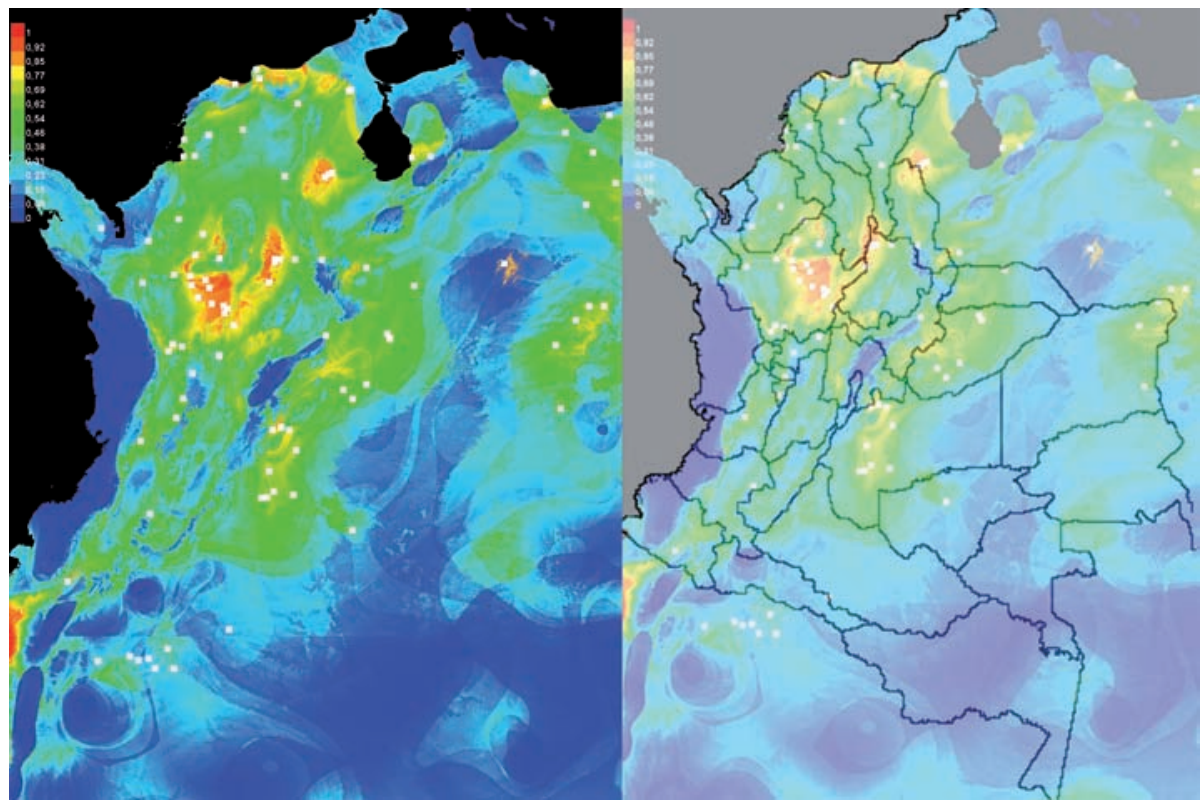

Figura 4. Modelo de nicho ecológico bajo las condiciones actuales para Alouatta seniculus en Colombia. Se destacan Antioquia, Bolívar, La Guajira, Magdalena, Meta, Santander, Norte de Santander y Vichada (elaborado con el programa Maxent, 2015)

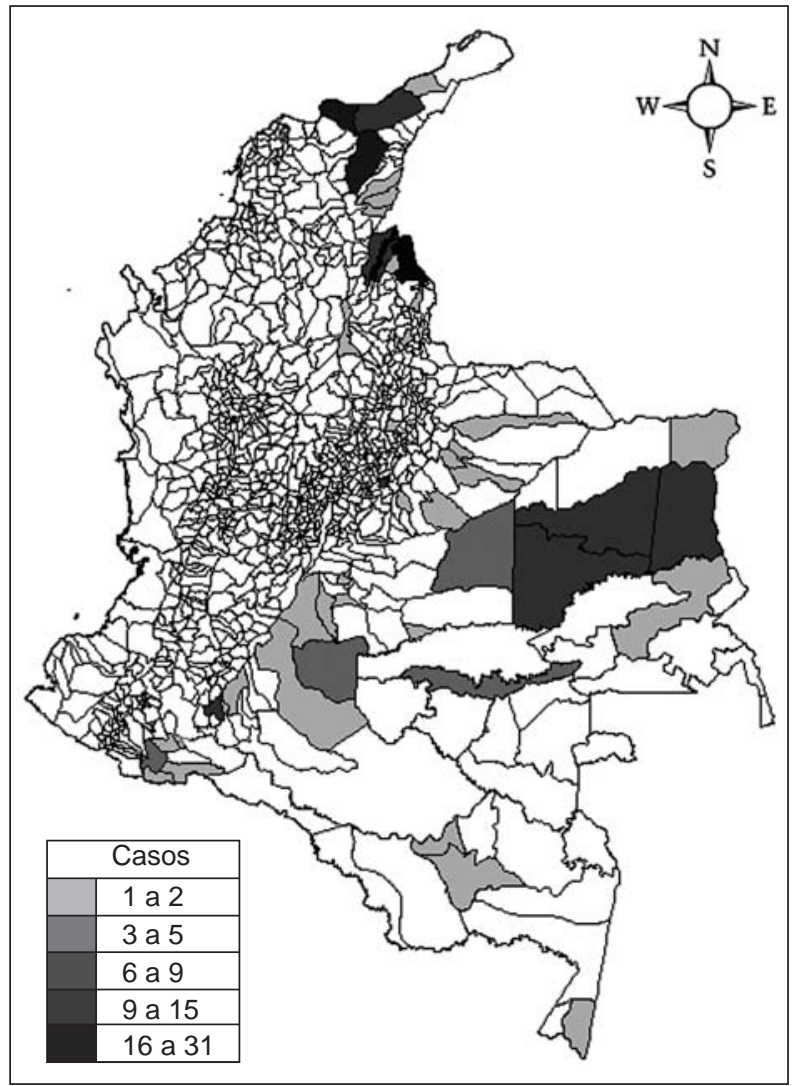

Figura 5. Municipios con casos confirmados de fiebre amarilla entre 2000 y 2009. Adaptado del informe de la enfermedad en el cuarto periodo epidemiológico, Instituto Nacional de Salud, 2015 (modelado con el programa DIVA-GIS) donde se encuentran las especies; no obstante, el análisis geográfico y de límites políticos en el país puede resultar más dispendioso (1). De cualquier modo, se sugiere tener en cuenta el trabajo de este y otros autores nacionales para futuras investigaciones.

Es fundamental establecer la coincidencia entre la presencia de primates no humanos y los antecedentes de fiebre amarilla, así como el modelo de nicho ecológico bajo las condiciones actuales, debido al papel que desempeñan dichos primates como reservorios naturales 0 huéspedes de la enfermedad. Sin embargo, es difícil elaborar una lista exhaustiva de los primates no humanos que pueden ser potenciales reservorios del virus de la fiebre amarilla, aunque se pueden puntualizar experiencias y aportes de entidades como la Organización Panamericana de Salud (OPS), los organismos del sector ambiental de países latinoamericanos, así como la información independiente, para determinar las especies de mayor distribución e importancia epidemiológica durante el ciclo de aparición de la fiebre amarilla selvática. Entre tales especies se destacan las del género Alouatta spp., que resulta involucrado en la mayoría de los casos de muerte reportados en primates no humanos en zonas con circulación comprobada del virus de la fiebre amarilla. Los dos aspectos clave para resaltar en este género son su amplia distribución 
Cuadro 1. Casos y muertes por fiebre amarilla (Segura, et al., 2013) (12) y presencia de Alouatta seniculus (GBIF, 2015)

\begin{tabular}{|c|c|c|c|c|}
\hline \multirow[t]{2}{*}{ Departamento o distrito } & \multicolumn{2}{|c|}{$\begin{array}{l}\text { Casos de fiebre amarilla } \\
2006 \text { a } 2009\end{array}$} & \multirow{2}{*}{$\begin{array}{c}\text { Muertes por fiebre } \\
\text { amarilla } \\
1998 \text { a } 2009\end{array}$} & \multirow{2}{*}{$\begin{array}{c}\text { Presencia de Alouatta seniculus } \\
\text { (según registros de GBIF y SiB } \\
\text { Colombia, 2015) }\end{array}$} \\
\hline & Probables & Confirmados & & \\
\hline Meta & 32 & 3 & 6 & 8 \\
\hline Vichada & 9 & 1 & 2 & 3 \\
\hline Bogotá & 6 & 0 & 1 & 0 \\
\hline Atlántico & 6 & 0 & 0 & 1 \\
\hline Caquetá & 5 & 1 & 5 & 1 \\
\hline Casanare & 4 & 0 & 3 & 5 \\
\hline Amazonas & 3 & 0 & 0 & 0 \\
\hline Putumayo & 2 & 0 & 1 & 0 \\
\hline Caldas & 2 & 0 & 1 & 2 \\
\hline Sucre & 2 & 0 & 0 & 3 \\
\hline Norte de Santander & 2 & 0 & 24 & 4 \\
\hline Huila & 1 & 0 & 0 & 0 \\
\hline Guainía & 1 & 0 & 1 & 0 \\
\hline Bolívar & 1 & 1 & 0 & 1 \\
\hline Tolima & 1 & 0 & 0 & 0 \\
\hline Boyacá & 1 & 0 & 1 & 1 \\
\hline Santander & 1 & 0 & 1 & 5 \\
\hline Guaviare & 1 & 0 & 2 & 1 \\
\hline Risaralda & 1 & 0 & 0 & 1 \\
\hline Antioquia & 1 & 0 & 0 & 17 \\
\hline Magdalena & 1 & 0 & 0 & 3 \\
\hline Cesar & 0 & 0 & 2 & 2 \\
\hline Vaupés & 0 & 0 & 1 & 0 \\
\hline Córdoba & 0 & 0 & 0 & 2 \\
\hline Chocó & 0 & 0 & 0 & 1 \\
\hline Valle & 0 & 0 & 0 & 1 \\
\hline Quindío & 0 & 0 & 0 & 1 \\
\hline Nariño & 0 & 0 & 0 & 1 \\
\hline Cauca & 0 & 0 & 0 & 1 \\
\hline Arauca & 0 & 0 & 0 & 1 \\
\hline La Guajira & 0 & 0 & 0 & 0 \\
\hline Cundinamarca & 0 & 0 & 0 & 0 \\
\hline San Andrés y Providencia & 0 & 0 & 0 & 0 \\
\hline
\end{tabular}

Cuadro 2. Reportes de casos confirmados de fiebre amarilla en primates no humanos en Colombia

\begin{tabular}{|c|c|c|c|c|c|}
\hline Especie & $\begin{array}{l}\text { Número de casos } \\
\text { confirmados/estudiados }\end{array}$ & $\begin{array}{l}\text { Método de } \\
\text { diagnóstico }\end{array}$ & $\begin{array}{l}\text { Año del } \\
\text { hallazgo }\end{array}$ & Lugar & Fuente \\
\hline Alouatta & $4 / 31$ & \multirow[t]{2}{*}{ Seroneutralización } & \multirow[b]{2}{*}{1979} & Bosques de & Vidales, et al. \\
\hline seniculus & $\begin{array}{l}\text { Otros cuatro esqueletos } \\
\text { observados en excursión }\end{array}$ & & & $\begin{array}{l}\text { Mariangola y Río } \\
\text { Seco, Cesar }\end{array}$ & $\begin{array}{l}\text { Biomédica } \\
1981 ; 4: 171-86\end{array}$ \\
\hline Alouatta & \multirow[t]{2}{*}{$1 / 1$} & \multirow[t]{2}{*}{ Sin datos } & \multirow[b]{2}{*}{2003} & \multirow{2}{*}{$\begin{array}{l}\text { Corregimiento } \\
\text { de Minca, } \\
\text { Santa Marta }\end{array}$} & Velandia, Revista \\
\hline $\begin{array}{l}\text { seniculus } \\
\text { rufiventris }\end{array}$ & & & & & $\begin{array}{l}\text { MVZ Córdoba. } \\
\text { 2004;9:459 }\end{array}$ \\
\hline Alouatta spp. & $4 / 5$ & $\begin{array}{l}\text { Inmunohistoquímica } \\
\text { y reacción en cadena } \\
\text { de la polimerasa }\end{array}$ & 2003 y 2004 & Cesar y Magdalena & $\begin{array}{l}\text { Méndez, et } \\
\text { al. Biomédica } \\
\text { 2007;27:461-7 }\end{array}$ \\
\hline \multirow[t]{2}{*}{ Alouatta spp. } & $2 / 2$ & \multirow[t]{3}{*}{ Aislamiento viral } & \multirow[b]{2}{*}{2003 y 2004} & \multirow{2}{*}{$\begin{array}{l}\text { Parque Ecológico } \\
\text { los Besotes, } \\
\text { Valledupar, Cesar }\end{array}$} & OPS/OMS \\
\hline & $\begin{array}{l}\text { Otros diez muertos de } \\
\text { tres manadas distintas }\end{array}$ & & & & $\begin{array}{l}\text { EER Noticias } \\
\text { Semanales } \\
\text { Vol. 2, No. 1, } 8 \\
\text { enero de } 2004\end{array}$ \\
\hline & & & & Parque Ecológico & INS. Boletín \\
\hline $\begin{array}{l}\text { Alouatta } \\
\text { seniculus }\end{array}$ & Sin datos & Inmunohistoquímica & 2004 & $\begin{array}{l}\text { los Besotes, } \\
\text { Valledupar, Cesar }\end{array}$ & $\begin{array}{l}\text { Epidemiológico } \\
\text { No. } 3,18 \text { a } 24 \text { de } \\
\text { enero de } 2004\end{array}$ \\
\hline
\end{tabular}


en selvas de Centroamérica y Suramérica, y su gran sensibilidad a pequeñas concentraciones de inoculación del virus $(3,4,13,14)$.

Otro aspecto importante en la definición de la importancia epidemiológica de los reservorios de la enfermedad, es reconocer que su presencia en las zonas selváticas de Colombia está condicionada por algunos factores ambientales que pueden modificar su distribución. Para referirse a la zoogeografía de las distintas especies y subespecies de primates no humanos en Colombia, es preciso describir el efecto de las diferencias altitudinales que se presentan en el país. Se sabe que el número de especies disminuye a medida que aumenta la altura sobre el nivel del mar, lo cual se debe al cambio de temperatura, cuyo gradiente térmico con relación a la altitud es de $0,55^{\circ} \mathrm{C}$ menos por cada 100 metros adicionales de altura.

Esas diferencias térmicas ejercen una gran presión en la supervivencia de los primates no humanos y se pueden explicar por dos aspectos fundamentales:

1) el efecto altitudinal implica consecuencias primarias sobre la diversidad de vegetales boscosos, que afecta, a su vez, la disponibilidad de alimento para los primates que allí habitan: a mayor altitud, menor disponibilidad de alimento;

2) el efecto primario de la altura y la temperatura sobre la tolerancia individual de la especie en cuestión implica que los pequeños primates no humanos que habitan Colombia tienen una tolerancia térmica restringida, es decir, pertenecen a especies estenotermas propias de rangos estrechos de temperatura que oscilan entre los 23 y los $28{ }^{\circ} \mathrm{C}$, muy sensibles a los cambios térmicos, por lo cual a mayor altitud y menor temperatura, menor también la tolerancia de los primates. Además, los aulladores requieren de zonas boscosas de estructura diversa para su desplazamiento y su alimentación $(1,15)$.

Dado que la vigilancia de la fiebre amarilla en Colombia no se hace en los reservorios, los mapas y la comprensión de la enfermedad se construyen a partir de los casos humanos. Se entiende que los sectores de salud y del ambiente son los responsables de generar ese conocimiento en el país, pero hoy no hay un sistema de vigilancia destinado a la detección y descripción del perfil epidemiológico de la enfermedad que recurra a sus reservorios naturales como centinelas, lo que permitiría fortalecer las acciones en salud humana y animal, así como priorizar zonas geográficas para su investigación y prevención $(5,13,16)$, además de consolidar los antecedentes de la enfermedad en el reservorio natural.

Considerando que algunos primates no humanos como $A$. seniculus son más propensos a la enfermedad, e históricamente se han relacionado más frecuentemente con la aparición de la fiebre amarilla, que su distribución depende de aspectos ambientales y que desempeñan un papel valioso como indicadores biológicos o centinelas de la circulación viral en zonas selváticas, el determinar la distribución geográfica de la enfermedad y de estos primates constituye un primer paso para establecer lineamentos de vigilancia epidemiológica, tal como lo señalan las experiencias de Argentina o Brasil, en donde se han elaborado protocolos para la vigilancia epidemiológica de epizootias de fiebre amarilla $(16,17)$.

La fiebre amarilla es una enfermedad zoonótica de transmisión vectorial. Los principales vectores que se encargan de su transmisión son $A$. aegypti y $A$. albopictus a nivel doméstico, y $H$. janthynomis y Sabethes sp. a nivel selvático. En Latinoamérica y en Colombia se lleva a cabo, asimismo, la vigilancia entomológica como apoyo a las decisiones en salud; en los protocolos de vigilancia de fiebre amarilla de países como Brasil, también se contempla la vigilancia en los vectores, para lo cual es importante generar el conocimiento sobre la presencia simultánea de la enfermedad, el reservorio y los vectores, con el fin de tener una visión más completa respaldada por el trabajo interdisciplinario de las áreas de medicina humana, médica veterinaria y otras ciencias biológicas para entender la ecología de las enfermedades transmitidas por vectores (18-20).

Algunos autores han planteado que el propósito de los sistemas de vigilancia en salud animal para el desarrollo de políticas en salud pública debe orientarse a formular hipótesis causales y determinar los factores de riesgo y los protectores asociados a la aparición de la enfermedad, pero también, a estudiar las características ambientales y los factores determinantes propios de la población de interés para llegar a conclusiones contextualizadas (20). Sin embargo, no hay estudios detallados sobre la presencia de los primates no humanos como un factor de riesgo para la fiebre amarilla en Colombia, lo cual va más allá de la descripción de epizootias en el curso de brotes de la enfermedad en humanos. Por otro lado, son más comunes los estudios que se basan en los análisis de riesgo y 
en la priorización de las poblaciones humanas que deben someterse a la vacunación que en el uso de los primates no humanos como centinelas de la circulación viral en el ámbito selvático $(5,15,17)$.

En otro estudio para determinar los factores de riesgo eco-entomológicos de la emergencia de arbovirus en zonas húmedas, se encontraron relaciones significativas en las frecuencias de cohabitación de vectores y reservorios. De cualquier modo, el estudio se centró en el virus del Nilo occidental y la presencia de aves con posibilidad de adquirirlo, aunque se ejemplificó la forma en que la coincidencia de huéspedes sensibles y vectores competentes puede representar un factor de riesgo para la emergencia o reemergencia de arbovirus en condiciones ambientales adecuadas (21).

En el 2007 se emitieron las guías de promoción de la salud y prevención de enfermedades en salud pública por parte del entonces Ministerio de la Protección Social de Colombia (22), en las cuales se determinó que los factores de riesgo propios de la enfermedad en humanos incluyen los siguientes: el ingreso a regiones enzoóticas sin vacunación; el tipo de ocupación, por ejemplo, en labores de tala de árboles (lo cual produce la reducción del número de mosquitos); el final de la época de lluvias cuando la densidad de los vectores es alta y la gente prepara las tierras para la siembra o la ganadería, y ser hombre entre los 15 y los 40 años de edad (los hombres se ven afectados cuatro veces más que las mujeres y suelen ocuparse más en el trabajo rural). Otros factores de riesgo de urbanización de la fiebre amarilla se relacionan con la expansión geográfica y el alto grado de infestación de las zonas urbanas por $A$. aegypti. El desplazamiento de la población por causa del conflicto armado que afecta el país también se estableció, entonces, como un factor de riesgo, pues favorece el asentamiento de poblaciones no vacunadas en áreas selváticas.

En el presente estudio se concluyó que, según los registros de la GBIF, en $69,5 \%$ de los 23 departamentos con antecedentes de fiebre amarilla había $A$. seniculus, es decir, que en $50 \%$ de los departamentos de Colombia había antecedentes de fiebre amarilla y registros de esta especie.

Los datos obtenidos de la GBIF son importantes porque no existe un conocimiento cabal sobre la distribución de los primates no humanos en Colombia, especialmente $A$. seniculus, ni de su relación con los antecedentes reportados de fiebre amarilla en el país. Aunque la información que se presenta no es definitiva, pues dependía del número de investigaciones y registros de la especie en Colombia en la plataforma consultada, el empleo de estas bases de datos es valioso para el estudio de temas de interés en salud pública.

Se sabe el papel que los primates no humanos desempeñan en la aparición de la fiebre amarilla, por lo que su empleo como centinelas de la circulación viral puede aportar enormemente a las estrategias de vigilancia de la enfermedad. Su relevancia como indicadores biológicos debe representar un incentivo para su estudio y protección, y para ampliar el conocimiento sobre la presencia simultánea de la enfermedad y el reservorio en el contexto de la formulación de nuevas investigaciones en salud y ambiente.

Debe incentivarse el trabajo interdisciplinario en medicina, medicina veterinaria y otras ciencias biológicas desde la perspectiva de la epidemiología y la salud pública, para entender mejor la ecología de las enfermedades transmitidas por vectores, así como para articular los sistemas de vigilancia en salud humana y animal, en especial de especies silvestres, a cargo de las autoridades de salud y ambiente.

\section{Agradecimientos}

A Pedro Enrique Navas-Suárez, del Departamento de Patología Comparativa y Experimental de la Universidad de São Paulo, Brasil; a Mauricio Javier Vera, del Ministerio de Salud y Protección Social de Colombia; a David Mauricio Suárez, diseñador gráfico de la Universidad Jorge Tadeo Lozano, Bogotá, y a Hernán David Maestre Piedrahita, diseñador industrial de la Pontificia Universidad Javeriana, Bogotá.

\section{Conflicto de intereses}

Los autores declaramos no tener conflicto de intereses en la realización de este artículo.

\section{Financiación}

El desarrollo del presente artículo no requirió de fuentes de financiación.

\section{Referencias}

1. Defler TR. Historia natural de los primates colombianos. Segunda edición. Bogotá: Universidad Nacional de Colombia; 2010. p. 324-34.

2. Rifakis PM, Benítez JA, De la Paz-Pineda J, RodríguezMorales AJ. Epizootics of yellow fever in Venezuela (20042005) an emerging zoonotic disease. Ann NY Acad Sci. 2006;1081:57-60. http://dx.doi.org/10.1196/annals.1373.005 
3. Auguste AJ, Lemey P, Pybus OG, Suchard MA, Salas RA, Adesiyun AA, et al. Yellow fever virus maintenance in Trinidad and its dispersal throughout the Americas. J Virol. 2010;84:9967-77. http://dx.doi.org/10.1128/JVI.00588-10

4. Lima MA, Romano-Lieber NS, Duarte AM. Circulation of antibodies against yellow fever virus in a simian population in the area of Porto Primavera Hydroelectric Plant, São Paulo, Brazil. Rev Inst Med Top São Paulo. 2010;52:11-5. http://dx.doi.org/10.1590/S0036-46652010000100002

5. Almeida MA, Cardoso J da C, Dos Santos E, da Fonseca DF, Cruz LL, Faraco FJ, et al. Surveillance for yellow fever virus in non-human primates in Southern Brazil, 2001-2011: A tool for prioritizing human populations for vaccination. PLoS Pathog. 2014;8:e2741. http://dx.doi.org/10.1371/ journal.pntd.0002741

6. Batista PM, Andreotti R, Almeida PS, Marques AC, Rodrigues SG, Chiang JO, et al. Detection of arboviruses of public health interest in free-living New World primates (Sapajus spp.; Alouatta caraya) captured in Mato Grosso do Sul, Brazil. Rev Soc Bras Med Trop. 2013;46:684-90. http:// dx.doi.org/10.1590/0037-8682-0181-2013

7. Câmara FP, Gomes AL, Carvalho LM, Castello LG. Dynamic behavior of sylvatic yellow fever in Brazil (19542008). Rev Soc Bras Med Trop. 2011;44:297-9. http://dx.doi. org/10.1590/S0037-86822011005000024

8. Méndez JA, Parra E, Neira M, Rey GJ. Detección por reacción en cadena de la polimerasa de transcriptasa inversa del virus de la fiebre amarilla en monos silvestres: una herramienta sensible para la vigilancia epidemiológica. Biomédica. 2007;7:461-7 http://dx.doi.org/10.7705/biomedica. v27i3.209

9. Velandia M. La reemergencia de la fiebre amarilla en Colombia, 2003. Revista MVZCórdoba. 2004;9:459.

10. Romano AP, Costa ZG, Ramos DG, Andrade MA, Jayme V de S, Almeida MA, et al. Yellow fever outbreaks in unvaccinated populations, Brazil, 2008-2009. PLoS Negl Trop Dis. 2014;8:e2740. http://dx.doi.org/10.1371/journal. pntd.0002740

11. Vasconcelos PF. Yellow fever in Brazil: Thoughts and hypotheses on the emergence in previously free areas. Rev Saúde Pública. 2010;44:1144-9. http://dx.doi.org/10.1590/ S0034-89102010005000046

12. Segura AM, Cardona D, Osley M. Tendencias de la mortalidad por fiebre amarilla, Colombia, 1998-2009. Biomédica. 2013;33(Supl.1):52-62. http://dx.doi.org/10. 7705/biomedica.v33i0.698
13. Tranquilin MV, Lehmkuhl RC, Maron A, Silva LR, Ziliotto L, Seki MC, et al. First report of yellow fever virus in nonhuman primates in the State of Paraná, Brazil. Rev Soc Bras Med Trop. 2013;46:522-4. http://dx.doi.org/10.1590/00378682-0106-2013

14. Carrington CV, Auguste AJ. Evolutionary and ecological factors underlying the tempo and distribution of yellow fever virus activity. Infect Genet Evol. 2013;13:198-210. http://dx.doi.org/10.1016/j.meegid.2012.08.015

15. Moreno ES, Spinola R, Tengan CH, Brasil RA, Siciliano MM, Coimbra TL, et al. Yellow fever epizootics in nonhuman primates, São Paulo state, Brazil, 2008-2009. Rev Inst Med Trop São Paulo. 2013;55:45-50. http://dx.doi. org/10.1590/S0036-46652013000100008

16. Moreno ES, Barata R de C. Methodology for definition of yellow fever priority areas, based on environmental variables and multiple correspondence analyses. PLoS Negl Trop Dis. 2012;6:e1658. http://dx.doi.org/10.1371/ journal.pntd.0001658.

17. Bicca-Marques JC, Freitas DS. The role of monkeys, mosquitoes, and humans in the occurrence of a yellow fever outbreak in a fragmented landscape in south Brazil: Protecting howler monkeys is a matter of public health. Trop Conserv Sci. 2010;3:78-89.

18. Bryant JE, Holmes EC, Barret AD. Out of Africa: A molecular perspective on the introduction of yellow fever virus into the Americas. PLoS Pathog. 2010;3:e75. http:// dx.doi.org/10.1371/journal.ppat.0030075

19. Gardner CL, Ryman KD. Yellow fever: A reemerging threat. Clin Lab Med. 2010;30:237-60. http://dx.doi.org/10.1016/j. cll.2010.01.001

20. Hoinville LJ, Alban L, Drewe JA, Gibbens JC, Gustafson J, Häsler B, et al. Proposed terms and concepts for describing and evaluating animal-health surveillance systems. Prev Vet Med. 2013;112:1-12. http://dx.doi.org/10.1016/j.prevetmed. 2013.06.006

21. Bañares AB, Marí RB, Chordá-Olmos FA, Jiménez-Peydro R. Factores de riesgo eco-entomológicos en la emergencia de arbovirosis en las zonas húmedas de la comunidad valenciana. Bol Mal Salud Amb. 2012;52:257-67.

22. Ministerio de la Protección Social. Guía 22. Guía de fiebre amarilla. En: Guías de Promoción de la Salud y Prevención de Enfermedades en la Salud Pública. Bogotá: Colección Programa de Apoyo a la Reforma de Salud (PARS), Ministerio de la Protección Social (MPS); 2007. p.227-45. 